\title{
A loss-based prior for variable selection in linear regression methods Supplementary Material
}

\author{
Cristiano Villa and Jeong Eun Lee
}

\section{Appendix}

\section{A Proof of Theorem 3.1}

Let $\gamma$ and $\gamma^{\prime}$ be non-identical binary vectors. With the corresponding design matrices $\mathbf{X}_{\boldsymbol{\gamma}}$ and $\mathbf{X}_{\gamma^{\prime}}$ and the vectors of coefficients $\beta_{\gamma}$ and $\beta_{\gamma^{\prime}}$, the models are defined as

$$
\begin{gathered}
M_{\boldsymbol{\gamma}}: \mathbf{y} \sim N_{n}\left(\mathbf{1}_{n} \alpha+\mathbf{X}_{\boldsymbol{\gamma}} \beta_{\boldsymbol{\gamma}}, \mathrm{I} / \phi\right)=N_{n}\left(\tilde{\mathbf{X}}_{\boldsymbol{\gamma}} \tilde{\beta}_{\gamma}, \mathrm{I} / \phi\right) \\
M_{\gamma^{\prime}}: \mathbf{y} \sim N_{n}\left(\mathbf{1}_{n} \alpha+\mathbf{X}_{\gamma^{\prime}} \beta_{\gamma^{\prime}}, \mathrm{I} / \phi\right)=N_{n}\left(\tilde{\mathbf{X}}_{\gamma^{\prime}} \tilde{\boldsymbol{\beta}}_{\gamma^{\prime}}, \mathrm{I} / \phi\right)
\end{gathered}
$$

where $\tilde{\mathbf{X}}_{\boldsymbol{\gamma}}=\left[\mathbf{1}, \mathbf{X}_{\boldsymbol{\gamma}}\right], \tilde{\mathbf{X}}_{\boldsymbol{\gamma}^{\prime}}=\left[\mathbf{1}, \mathbf{X}_{\boldsymbol{\gamma}^{\prime}}\right], \tilde{\beta}_{\boldsymbol{\gamma}}=\left(\alpha, \beta_{\gamma}\right)^{T}$, and $\tilde{\beta}_{\gamma^{\prime}}=\left(\alpha, \beta_{\gamma^{\prime}}\right)^{T}$. The Kullback-Leibler divergence of $M_{\gamma}$ from $M_{\gamma^{\prime}}$ is

$$
\begin{aligned}
& D_{K L}\left(M_{\boldsymbol{\gamma}} \| M_{\gamma^{\prime}}\right) \\
& =\int m\left(\mathbf{y} \mid M_{\gamma}\right)\left\{-\frac{\log (1 / \phi)}{2}-\frac{\phi}{2}\left(\mathbf{y}-\tilde{\mathbf{X}}_{\gamma} \tilde{\beta}_{\gamma}\right)^{T}\left(\mathbf{y}-\tilde{\mathbf{X}}_{\gamma} \tilde{\beta}_{\gamma}\right)+\frac{\log (1 / \phi)}{2}\right. \\
& \left.+\frac{\phi}{2}\left(\mathbf{y}-\tilde{\mathbf{X}}_{\gamma^{\prime}} \tilde{\boldsymbol{\beta}}_{\gamma^{\prime}}\right)^{T}\left(\mathbf{y}-\tilde{\mathbf{X}}_{\gamma^{\prime}} \tilde{\boldsymbol{\beta}}_{\gamma^{\prime}}\right)\right\} d \mathbf{y} \\
& =\int m\left(\mathbf{y} \mid M_{\gamma}\right) \phi\left\{-\frac{1}{2} \mathbf{y}^{T} \mathbf{y}+\left(\tilde{\mathbf{X}}_{\gamma} \tilde{\beta}_{\gamma}\right)^{T} \mathbf{y}-\frac{1}{2}\left(\tilde{\mathbf{X}}_{\gamma} \tilde{\beta}_{\gamma}\right)^{T}\left(\tilde{\mathbf{X}}_{\gamma} \tilde{\beta}_{\gamma}\right)+\frac{1}{2} \mathbf{y}^{T} \mathbf{y}-\left(\tilde{\mathbf{X}}_{\gamma^{\prime}} \tilde{\boldsymbol{\beta}}_{\gamma^{\prime}}\right)^{T} \mathbf{y}\right. \\
& \left.+\frac{1}{2}\left(\tilde{\mathbf{X}}_{\boldsymbol{\gamma}^{\prime}} \tilde{\boldsymbol{\beta}}_{\boldsymbol{\gamma}^{\prime}}\right)^{T}\left(\tilde{\mathbf{X}}_{\boldsymbol{\gamma}^{\prime}} \tilde{\boldsymbol{\beta}}_{\boldsymbol{\gamma}^{\prime}}\right)\right\} d \mathbf{y} \\
& =\frac{\phi}{2}\left(\left(\tilde{\mathbf{X}}_{\boldsymbol{\gamma}} \tilde{\boldsymbol{\beta}}_{\boldsymbol{\gamma}}\right)^{T}\left(\tilde{\mathbf{X}}_{\boldsymbol{\gamma}} \tilde{\boldsymbol{\beta}}_{\boldsymbol{\gamma}}\right)-2\left(\tilde{\mathbf{X}}_{\boldsymbol{\gamma}^{\prime}} \tilde{\boldsymbol{\beta}}_{\boldsymbol{\gamma}^{\prime}}\right)^{T}\left(\tilde{\mathbf{X}}_{\boldsymbol{\gamma}} \tilde{\boldsymbol{\beta}}_{\boldsymbol{\gamma}}\right)+\left(\tilde{\mathbf{X}}_{\boldsymbol{\gamma}^{\prime}} \tilde{\boldsymbol{\beta}}_{\boldsymbol{\gamma}^{\prime}}\right)^{T}\left(\tilde{\mathbf{X}}_{\boldsymbol{\gamma}^{\prime}} \tilde{\boldsymbol{\beta}}_{\boldsymbol{\gamma}^{\prime}}\right)\right)
\end{aligned}
$$

The first derivative of $D_{K L}\left(M_{\gamma} \| M_{\gamma^{\prime}}\right)$ with respect to $\tilde{\beta}_{\gamma^{\prime}}$ is

$$
\frac{\partial D_{K L}\left(M_{\boldsymbol{\gamma}} \| M_{\boldsymbol{\gamma}^{\prime}}\right)}{\partial \tilde{\boldsymbol{\beta}}_{\boldsymbol{\gamma}^{\prime}}}=\phi\left(-\left(\tilde{\mathbf{X}}_{\boldsymbol{\gamma}} \tilde{\boldsymbol{\beta}}_{\boldsymbol{\gamma}}\right)^{T}+\left(\tilde{\mathbf{X}}_{\boldsymbol{\gamma}^{\prime}} \tilde{\boldsymbol{\beta}}_{\gamma^{\prime}}\right)^{T}\right) \tilde{\mathbf{X}}_{\boldsymbol{\gamma}^{\prime}} .
$$

If $\tilde{\mathbf{X}}_{\gamma^{\prime}} \tilde{\mathbf{X}}_{\gamma^{\prime}}^{T}$ is invertible, $D_{K L}\left(M_{\gamma} \| M_{\gamma^{\prime}}\right)$ is minimized when $\tilde{\boldsymbol{\beta}}_{\gamma^{\prime}}=\left(\tilde{\mathbf{X}}_{\gamma^{\prime}}^{T} \tilde{\mathbf{X}}_{\gamma^{\prime}}\right)^{-1} \tilde{\mathbf{X}}_{\gamma^{\prime}}^{T} \tilde{\mathbf{X}}_{\gamma} \tilde{\boldsymbol{\beta}}_{\boldsymbol{\gamma}}$ and the minimum is given by

$$
\min _{\tilde{\beta}_{\gamma^{\prime}}} D_{K L}\left(M_{\gamma} \| M_{\gamma^{\prime}}\right)=0
$$




\section{$B$ Details of the simulations studies - Noninformative prior}

In this Section we present the detailed results of the simulation studies carried on in Section 4.1 of the paper.

In particular, for the Scott and Berger prior and the loss-based prior obtained with the three proposed methods to calibrate $c$, we report the coverage of the posterior $95 \%$ credible interval, the posterior mean squared error from the mean and the frequency at which the true model is identified by the HPM.

Table A.1 reports the results when a sample size of $n=50$ has been used, while Table A.2 report the results for the case $n=100$.

\begin{tabular}{cccccccccccccc}
\hline & & S\&B & \multicolumn{1}{c}{ LB-LD } & \multicolumn{1}{c}{ LB-ML } & \multicolumn{1}{c}{ LB-GB } \\
$d$ & COV & MSE & FQT & COV & MSE & FQT & COV & MSE & FQT & COV & MSE & FQT & $\omega$ \\
\hline 5 & 1.000 & 0.355 & 0.860 & 0.996 & 0.139 & 0.868 & 0.996 & 0.194 & 0.904 & 0.992 & 0.623 & 0.800 & 0.05 \\
& 1.000 & 0.413 & 0.748 & 0.992 & 0.232 & 0.752 & 1.000 & 0.215 & 0.828 & 0.992 & 0.516 & 0.660 & 0.15 \\
& 0.996 & 0.483 & 0.688 & 0.980 & 0.378 & 0.636 & 0.976 & 0.402 & 0.656 & 1.000 & 0.410 & 0.604 & 0.25 \\
& 0.992 & 0.580 & 0.480 & 0.876 & 0.969 & 0.448 & 0.924 & 0.746 & 0.476 & 0.948 & 0.595 & 0.480 & 0.50 \\
& 0.984 & 0.791 & 0.364 & 0.796 & 1.587 & 0.296 & 0.828 & 1.207 & 0.364 & 0.788 & 1.528 & 0.224 & 0.75 \\
10 & 1.000 & 0.486 & 0.896 & 1.000 & 0.151 & 0.920 & 0.996 & 0.464 & 0.852 & 0.960 & 2.072 & 0.728 & 0.05 \\
& 1.000 & 0.497 & 0.888 & 1.000 & 0.115 & 0.892 & 1.000 & 0.256 & 0.872 & 1.000 & 1.211 & 0.788 & 0.15 \\
& 0.992 & 0.732 & 0.784 & 0.972 & 0.260 & 0.780 & 1.000 & 0.290 & 0.728 & 1.000 & 0.410 & 0.604 & 0.25 \\
& 1.000 & 0.973 & 0.608 & 0.920 & 0.825 & 0.536 & 0.976 & 0.516 & 0.556 & 0.984 & 0.514 & 0.612 & 0.50 \\
& 0.996 & 0.739 & 0.352 & 0.892 & 0.919 & 0.316 & 0.852 & 0.952 & 0.340 & 0.880 & 1.065 & 0.404 & 0.75 \\
15 & 1.000 & 0.364 & 0.952 & 1.000 & 0.116 & 0.964 & 0.992 & 0.960 & 0.880 & 0.976 & 2.088 & 0.872 & 0.05 \\
& 1.000 & 0.351 & 0.820 & 0.992 & 0.137 & 0.860 & 1.000 & 0.320 & 0.852 & 0.988 & 1.066 & 0.888 & 0.15 \\
& 1.000 & 0.462 & 0.744 & 0.956 & 0.413 & 0.744 & 1.000 & 0.313 & 0.760 & 1.000 & 0.515 & 0.864 & 0.25 \\
& 1.000 & 1.001 & 0.512 & 0.968 & 0.524 & 0.352 & 0.908 & 0.102 & 0.840 & 0.980 & 0.953 & 0.544 & 0.50 \\
& 1.000 & 1.054 & 0.200 & 0.820 & 1.325 & 0.480 & 0.788 & 0.115 & 0.524 & 0.880 & 0.967 & 0.472 & 0.75 \\
30 & 1.000 & 0.249 & 0.928 & 0.996 & 0.105 & 0.920 & 1.000 & 0.012 & 0.988 & 0.972 & 1.809 & 0.916 & 0.05 \\
& 1.000 & 0.593 & 0.696 & 0.948 & 0.502 & 0.632 & 0.988 & 0.148 & 0.848 & 1.000 & 0.613 & 0.928 & 0.15 \\
& 1.000 & 1.020 & 0.428 & 0.948 & 0.272 & 0.316 & 0.940 & 0.350 & 0.744 & 1.000 & 0.463 & 0.844 & 0.25 \\
& 1.000 & 1.714 & 0.152 & 0.884 & 1.008 & 0.484 & 0.848 & 1.583 & 0.444 & 0.980 & 0.558 & 0.584 & 0.50 \\
& 1.000 & 2.767 & 0.048 & 0.565 & 2.904 & 0.244 & 0.320 & 3.012 & 0.172 & 0.736 & 2.385 & 0.256 & 0.75 \\
\hline
\end{tabular}

TABLE A.1

Summary of simulation results for the (noniformative) priors with $n=50$ : Scott and Berger prior (SEBB) Loss-based prior with $c=\log (d)$ (SB-lg), Loss-based prior with $c=1.2785$ (LB-ml) and hierarchical loss-based prior (LB-gb). A proportion of identifying the true number of covariates within the $95 \%$ of the posterior probability (COV), the mean squared error of posterior mean values (MSE) and the frequency the true model (FQT) is identified (by the HPM) are computed.

To ease the interpretation of the frequency that the true model has been identified by the HPM (the FQT index in the tables), we have included Figure A.1 and Figure A.2 to represent, under each considered prior, the above frequency for the cases, respectively, $n=50$ and $n=100$. 


\begin{tabular}{cccccccccccccc}
\hline & & S\&B & \multicolumn{1}{c}{ LB-LD } & \multicolumn{1}{c}{ LB-ML } & \multicolumn{3}{c}{ LB-GB } \\
$d$ & COV & MSE & FQT & COV & MSE & FQT & COV & MSE & FQT & COV & MSE & FQT & $\omega$ \\
\hline 5 & 0.988 & 0.196 & 0.928 & 0.996 & 0.096 & 0.932 & 0.992 & 0.100 & 0.912 & 0.996 & 0.512 & 0.808 & 0.05 \\
& 1.000 & 0.230 & 0.828 & 1.000 & 0.153 & 0.836 & 0.996 & 0.183 & 0.840 & 0.992 & 0.395 & 0.804 & 0.15 \\
& 0.992 & 0.373 & 0.736 & 0.992 & 0.221 & 0.744 & 0.980 & 0.228 & 0.792 & 0.988 & 0.308 & 0.760 & 0.25 \\
& 0.996 & 0.500 & 0.524 & 0.948 & 0.557 & 0.564 & 0.920 & 0.752 & 0.588 & 0.976 & 0.392 & 0.572 & 0.50 \\
& 0.980 & 0.643 & 0.472 & 0.876 & 0.942 & 0.452 & 0.872 & 0.952 & 0.416 & 0.876 & 0.839 & 0.432 & 0.75 \\
10 & 1.000 & 0.218 & 0.960 & 1.000 & 0.065 & 0.976 & 0.996 & 0.232 & 0.908 & 0.976 & 1.971 & 0.756 & 0.05 \\
& 0.992 & 0.426 & 0.864 & 0.996 & 0.138 & 0.884 & 0.996 & 0.276 & 0.828 & 0.992 & 1.172 & 0.784 & 0.15 \\
& 1.000 & 0.520 & 0.788 & 0.984 & 0.234 & 0.784 & 1.000 & 0.236 & 0.832 & 0.988 & 0.873 & 0.832 & 0.25 \\
& 0.996 & 0.912 & 0.676 & 0.968 & 0.359 & 0.676 & 0.964 & 0.438 & 0.720 & 0.992 & 0.345 & 0.696 & 0.50 \\
& 1.000 & 0.741 & 0.460 & 0.924 & 0.742 & 0.504 & 0.924 & 0.822 & 0.460 & 0.876 & 0.830 & 0.520 & 0.75 \\
15 & 1.000 & 0.201 & 0.956 & 1.000 & 0.078 & 0.956 & 0.992 & 0.412 & 0.936 & 0.984 & 2.020 & 0.808 & 0.05 \\
& 1.000 & 0.227 & 0.920 & 1.000 & 0.081 & 0.912 & 1.000 & 0.148 & 0.920 & 0.996 & 1.049 & 0.936 & 0.15 \\
& 1.000 & 0.333 & 0.856 & 0.980 & 0.222 & 0.800 & 1.000 & 0.188 & 0.852 & 0.992 & 1.040 & 0.852 & 0.25 \\
& 1.000 & 0.666 & 0.696 & 0.908 & 0.645 & 0.580 & 0.980 & 0.499 & 0.632 & 0.992 & 0.442 & 0.708 & 0.50 \\
& 1.000 & 0.824 & 0.540 & 0.884 & 0.756 & 0.572 & 0.956 & 0.714 & 0.520 & 0.896 & 0.986 & 0.508 & 0.75 \\
30 & 1.000 & 0.142 & 0.980 & 0.996 & 0.183 & 0.984 & 1.000 & 0.012 & 0.988 & 0.968 & 1.784 & 0.932 & 0.05 \\
& 1.000 & 0.334 & 0.812 & 0.984 & 0.232 & 0.776 & 1.000 & 0.444 & 0.784 & 0.996 & 0.689 & 0.960 & 0.15 \\
& 1.000 & 0.640 & 0.676 & 0.916 & 0.667 & 0.584 & 1.000 & 0.408 & 0.684 & 1.000 & 0.471 & 0.884 & 0.25 \\
& 1.000 & 1.373 & 0.316 & 0.952 & 0.538 & 0.628 & 0.923 & 0.888 & 0.568 & 0.992 & 0.894 & 0.332 & 0.50 \\
& 1.000 & 2.212 & 0.224 & 0.844 & 1.192 & 0.448 & 0.484 & 1.972 & 0.352 & 0.884 & 0.958 & 0.448 & 0.75 \\
\hline
\end{tabular}

TABLE A.2

Summary of simulation results for the (noninformative) priors with $n=50:$ Scott and Berger prior (SEB ) Loss-based prior with $c=\log (d)$ (SB-lg), Loss-based prior with $c=1.2785$ (LB-ml) and hierarchical loss-based prior (LB-gb). A proportion of identifying the true number of covariates within the $95 \%$ of the posterior probability (COV), the mean squared error of posterior mean values (MSE) and the frequency the true model (FQT) is identified (by the HPM) are computed.
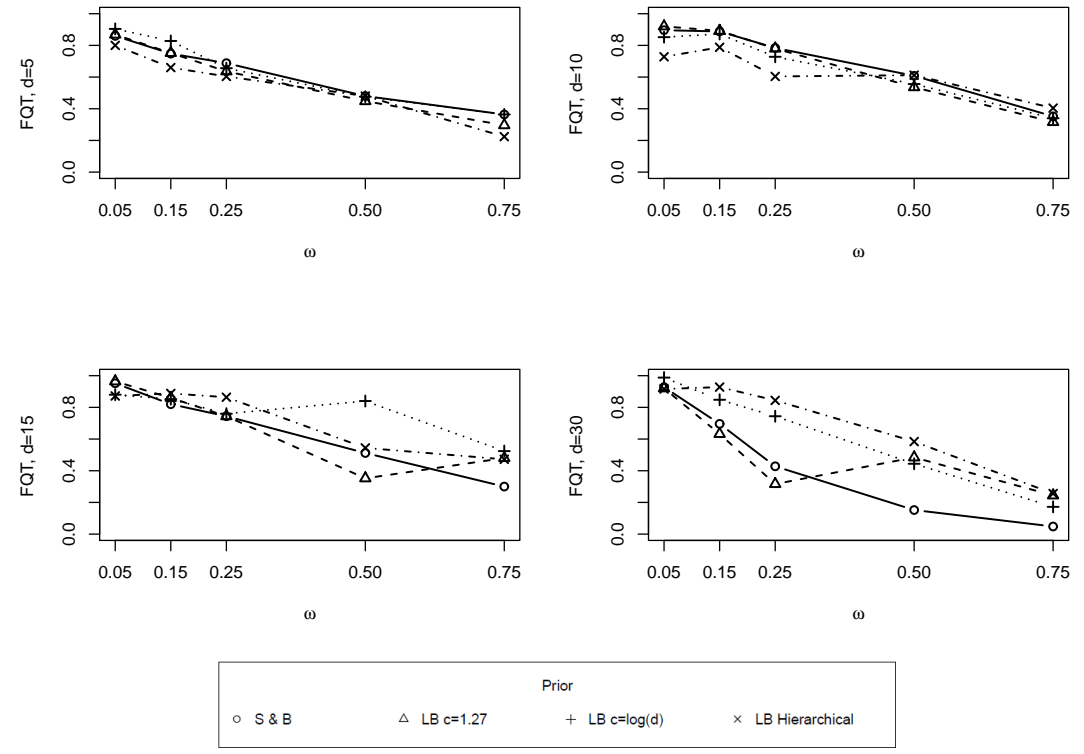

FIG A.1. Frequency of the true model (FQT), as identified by the HPM, under the Scott and Berger prior and the loss-based prior, for the three methods of calibration of $c$. The plots represent the posterior summary statistic for different values of $d$ and for $n=50$. 

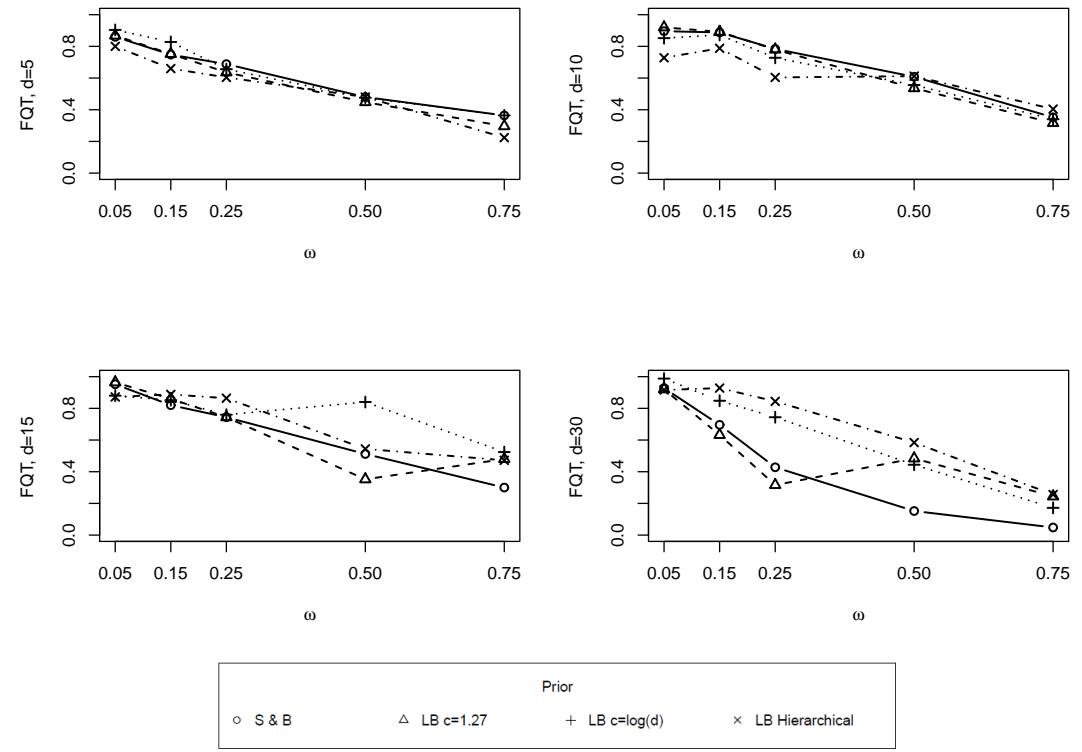

FIG A.2. Frequency of the true model (FQT), as identified by the HPM, under the Scott and Berger prior and the loss-based prior, for the three methods of calibration of c. The plots represent the posterior summary statistic for different values of $d$ and for $n=100$.

\section{Details of the simulations studies - Informative prior}

In this Appendix we report some details of the simulation study described in Section 4.2 of the paper. In particular, Figures A.3 and A.4 show the frequentist results, in the form of violin plots, of the simulation study. 


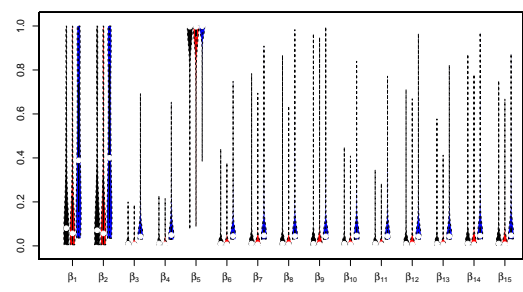

(a)

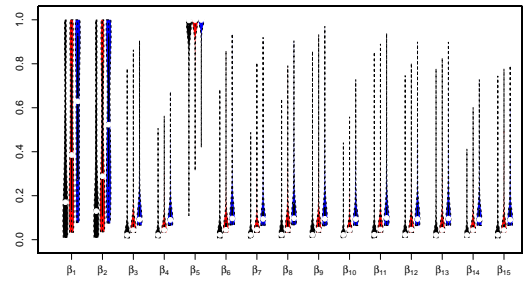

(c)

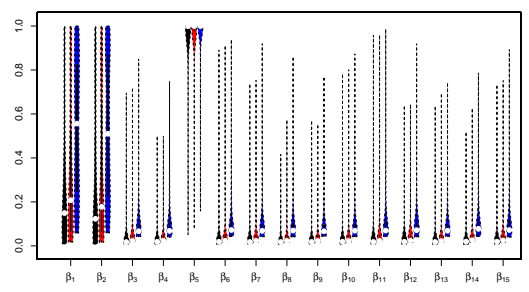

(b)

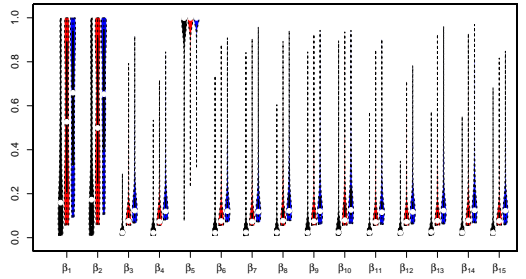

(d)

FIG A.3. $P\left(\beta_{i} \neq 0 \mid y\right)$ for $d=15, n=50$ and $\beta=(0.5,-0.5,0,0,-1)$ and an additional 10 null coefficeints. Comparison of the Beta-Binomial prior (left black violin) the loss-based prior with fixed $c$ (middle red violin) and the loss based prior with the informative hierarchical Generalised Beta (right blue violin) with prior mean equal to 1 (a), equal to 3 (b), equal to 5 (c) and equal to $7(d)$. The empty circles show the mean. Each violin represnts the distribution (approximated by a kernel function) of the posterior inclusion probability over the repeated samples. 


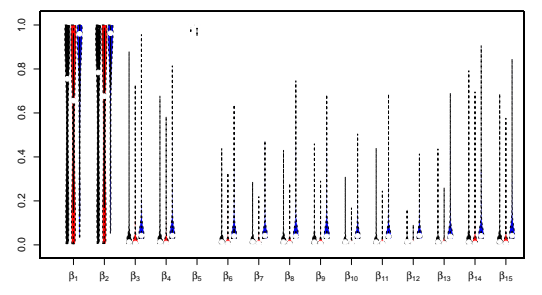

(a)

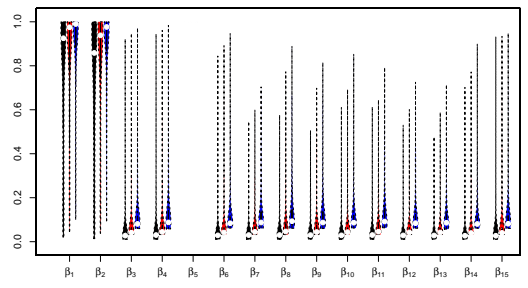

(c)

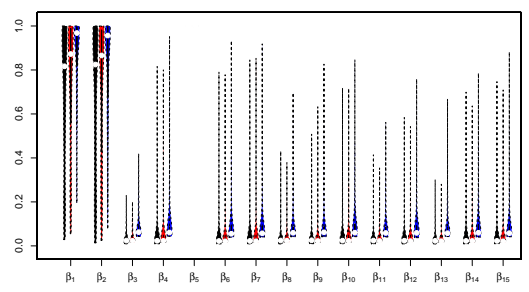

(b)

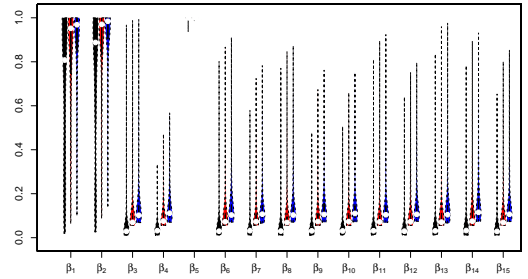

(d)

FIG A.4. $P\left(\beta_{i} \neq 0 \mid y\right)$ for $d=15, n=100$ and $\beta=(0.5,-0.5,0,0,-1)$ and an additional 10 null coefficeints. Comparison of the Beta-Binomial prior (left black violin) the loss-based prior with fixed $c$ (middle red violin) and the loss based prior with the informative hierarchical Generalised Beta (right blue violin) with prior mean equal to 1 (a), equal to 3 (b), equal to 5 (c) and equal to 7 (d). The empty circles show the mean. Each violin represnts the distribution (approximated by a kernel function) of the posterior inclusion probability over the repeated samples. 